\title{
LA TRIANGULACIÓN MÚLTIPLE EN LA INVESTIGACIÓN-ACCIÓN CON EJEMPLOS DE INVESTIGACIÓN PROPIA
}

\section{Triangulation in action research on the basis of own investigation}

The aim of the article is to reflect upon the possibilities offered by triangulation in qualitative research, with particular emphasis placed on action research. Various types of triangulation have been presented, including the most recommended multiple triangulation. A variety of advantages and functions of triangulation have been shown, first and foremost the ones which refer to dynamic, systemic and multicausal phenomena. All the theoretical considerations have been illustrated by the author's own research conducted in Poland, in one of the bilingual sections with Spanish language in the secondary school. The research concerned the development of students' discursive competence within the genre of an intercultural comparison, and its conditioning. The research used multiple triangulation: of data sources, of researchers, of methods and of data analysis, as well as temporal and special triangulation. The investigation is finished with conclusions of methodological nature.

Keywords: triangulation, action research, qualitative investigation, CLIL, discursive competence, Spanish as a foreign language, student's autonomy, intercultural comparisons

Słowa kluczowe: triangulacja, badanie w działaniu, badania jakościowe, CLIL, kompetencja dyskursywna, hiszpański jako język obcy, autonomia ucznia, porównania interkulturowe 


\section{Introducción}

El primer objetivo del presente artículo es reflexionar sobre las posibilidades de triangulación en la investigación-acción. El segundo, y quizás más importante, es presentar las diferentes perspectivas que aporta a una misma investigación el uso de diferentes tipos de triangulación, teniendo como punto de partida y ejemplo la investigación propia. Al final intentaremos sacar una serie de conclusiones de naturaleza más general.

\section{La triangulación en investigación-acción}

En el presente texto se entenderá la investigación-acción como un método cualitativo de investigación que consiste en detectar un problema o reto en la práctica educativa, preparar una intervención fundamentada teóricamente a pequeña escala en el funcionamiento de un grupo reducido de personas, ponerla en práctica por el propio investigador y analizar sus efectos (más detalles sobre la investigación-acción y sus etapas véase: Burns 1999, Pilch, Bauman 2001, Aleksandrzak 2004, Wiśniewska 2009, Kemmis, McTaggart 2009). El término investigación-acción incluye también la situación, sin duda muy valiosa, en la que los fines son sobre todo prácticos y la investigación la realizan profesores con capacidad de reflexión pero que no son científicos. Estas situaciones quedan fuera de nuestro interés. La investigación que se describirá en adelante forma parte de una tesis doctoral no publicada (Majewska, 2013) y la persona que la ha realizado era en aquel entonces profesora de secundaria y doctoranda a la vez.

La triangulación es un término de origen náutico y de geodesia y se refiere a la toma de dos puntos (perspectivas diferentes) para establecer la posición de un objeto. En la metodología, el término fue introducido por D. Campbell y D. Fiske en 1959 (Urbaniak-Zając 2018), quienes observaron que el uso de diferentes métodos permite minimizar el error resultante del uso de una herramienta de investigación para la validación de dicha investigación. Proponen alcanzar la fiabilidad por medio del uso de instrumentos parecidos (solo cualitativos o solo cuantitativos) y la validez gracias a recurrir a multimétodos, combinando los instrumentos de índole cualitativa y cuantitativa (Alzás García et al. 2016, Urbaniak-Zając 2018).

El teórico de la triangulación más citado es N. Denzin, representante de la metodología cualitativa, quien entendió la triangulación como una estrategia metodológica que consiste en aprovechar procedimientos de investigación paralelos (Denzin y Lincoln (reds.) 2009:26). El objetivo de la triangulación es conseguir la validez de la investigación y su completez. N. Denzin ya en el año 
1970 propuso distinguir cuatro tipos de triangulación: (1) de fuentes de datos, (2) de investigadores, (3) teórica y (4) metodológica.

La triangulación de fuentes de datos da la posibilidad de contrastar los datos de fuentes diversas y es la más frecuente en la investigación social (Alzás García et al. 2016). Puede realizarse investigando varios grupos (triangulación personal), en diferentes lugares (triangulación espacial) y en diferentes momentos de tiempo (triangulación temporal) (Aguilar, Barroso, 2015: 74, Hornowska et al. 2012: 74). La triangulación de investigadores, como el nombre mismo indica, consiste en realizar la investigación por más de un investigador (pueden ser equipos multidisciplinares) evitando de este modo el riesgo de sesgo. La triangulación teórica es la menos usada, requiere el uso de distintas teorías que pueden ser hasta antagónicas para llegar a una mayor comprensión de un mismo fenómeno. La triangulación metodológica puede consistir en el uso de un método, pero con distintas técnicas de recogida de datos y su posterior análisis (triangulación intramétodo) o en la combinación de varias metodologías, por ejemplo los métodos cualitativos y cuantitativos (triangulación intermétodo).

En las fuentes teóricas sobre la metodología podemos encontrar también las siguientes denominaciones de tipos de triangulación: la de diferentes disciplinas, de fuentes de datos, de datos, de maneras de analizar los datos, de estrategias de investigación, de métodos de investigación, de tipos de investigación, de perspectivas de investigación, de creación de temas, construcción de conocimientos y evaluación de resultados (véase Flick 2007, Szymańska et al. 2018). En nuestra opinión, las más importantes siguen siendo las de Denzin y una más, a saber, la triangulación en el análisis que consiste en el "uso de dos o más aproximaciones en el análisis de un mismo grupo de datos para propósitos de validación" (Arias Valencia 2000: 20).

Existe también la posibilidad de hacer uso de los varios tipos de triangulación enumerados por Denzin en una misma investigación (p. ej. de datos, de investigadores, metodológica y / o teórica). Este investigador propone el nombre de la triangulación multimétodos para denominar el uso de por lo menos dos de los tipos de triangulación indicados (Alzás García 2016). Otros investigadores prefieren el nombre de triangulación múltiple para el mismo fenómeno indicando que "se basa en usar más de un nivel de análisis" (Aguilar y Barroso, 2015: 75). En nuestra investigación optamos por el nombre de triangulación múltiple ya que la denominación multimétodos, a nuestro modo de ver, sugiere referirse solo a un tipo de triangulación, la metodológica y la múltiple hace hincapié claramente en el número y variedad de sus tipos.

Hay que subrayar que la triangulación es una estrategia integradora desde el punto de vista metodológico, y no solo una estrategia aditiva. La integración se consigue dando una perspectiva nueva que permita entender 
más profundamente y explicar mejor el fenómeno investigado. Por consiguiente, no se puede hablar de una triangulación cuando se recogen muchos datos irrelevantes o de poco valor para interpretar el fenómeno en cuestión. Tampoco si no se comparte "un mismo objeto de investigación en su diseño, procedimiento y análisis de los resultados" (Alzás García et al. 2016: 643).

La triangulación, y sobre todo la múltiple, si se aplica bien, puede aportar muchos beneficios a la investigación.

En primer lugar, la triangulación es un procedimiento fundamental en la investigación cualitativa, en la que no se analiza la muestra representativa, lo cual dificulta la fiabilidad y la validez. Según K. Konarzewski (2000), por esta razón la ampliación y consolidación de conocimientos parecen ser más importantes en la investigación cualitativa que la validez. Se relacionan directamente con la apertura a conocimientos nuevos y la reducción de sesgo. E. Hornowska y otros (2012: 75) analizan el concepto de la validez en la triangulación y constatan que la validez convergente, según la cual diferentes métodos que miden el mismo fenómeno deberían dar resultados parecidos, solo puede aplicarse con éxito a "fenómenos tratados como estáticos, unicausales y que no se perciben como partes de sistemas", o sea, que no tiene razón de ser cuando se trata de conocer la dinámica de un proceso, de un fenómeno que forma parte de un sistema, de un fenómeno que puede tener y tiene causas múltiples y cuando el objetivo es la descripción holística de este fenómeno. Estamos completamente de acuerdo con esta constatación. Este será el caso de nuestra investigación.

Además, la validez no se puede conseguir si no se presentan los criterios de selección de los casos a investigar ni tampoco cuando no se indican las fuentes de datos originales (sino únicamente las citas elegidas) y si se exponen solo algunos casos explicativos (Silverman, 2008: 273). Mientras que las primeras dos condiciones se han cumplido en nuestro estudio, la última exigencia ha sido imposible de cumplir. Ocurre que a veces es difícil de realizar, ya que en la realidad educativa ocurren fenómenos muy marcados por el contexto, excepcionales o característicos para un momento determinado, por lo que es complicado encontrar una prueba concluyente o repetir la investigación. Es un rasgo característico de todas las investigaciones cualitativas en la pedagogía, en las que "la explicación es posible solo para un lugar y tiempo, no es posible generalizar las explicaciones a toda la población y a diferentes momentos en el tiempo" (Palka 2006: 54).

Respecto a la fiabilidad, muchos investigadores afirman que con la triangulación aumenta la fiabilidad de la investigación que se refiere "al grado de certeza con la que podemos fiarnos de las fuentes de datos y, en consecuencia, de los datos basados en estas fuentes" (Hornowska et al. 2012: 75). 
Se puede decir que en realidad sus indicadores serían sobre todo la similitud o la semejanza de los datos obtenidos. Pero, como se ha indicado, si el proceso es dinámico y muchos factores influyen en él, no se puede esperar siempre los mismos resultados. Por otro lado y por esa misma razón, la obtención de resultados diferentes con el mismo instrumento de investigación no siempre tiene que significar que el instrumento no sea fiable.

En general, con los procesos dinámicos, sistémicos y multicausales la búsqueda de una sola, clara y unívoca imagen del fenómeno investigado es un objetivo un tanto ingenuo (Hornowska et al 2012: 75). La realidad educativa es multifacética y puede dar muchas imágenes igual de válidas en sus respectivos contextos. Los datos contradictorios nos pueden indicar que el fenómeno tiene un carácter único y excepcional, si bien sabemos que las excepciones también forman parte de la realidad humana. Nos pueden indicar que la relación entre los fenómenos es diferente de la que suele suceder en la mayoría de los casos. De este modo podemos descubrir lo que no es evidente, pero sí que es real y ocurre. La triangulación nos permite describir los fenómenos de manera más detallada, desde diferentes puntos de vista, nos permite percibir la complejidad de un sistema dinámico. Todo eso nos lleva al entendimiento más profundo de la realidad investigada. El comportamiento humano rara vez es explicable en términos de una simple relación de causa-efecto. Por el contrario, es sumamente complicado detectar las posibles causas de las decisiones y los efectos de la actuación humana.

Todos los investigadores están de acuerdo en que para que la triangulación aporte los beneficios deseados, es necesario un rigor metodológico constante, mucha precisión, la evaluación constante de los instrumentos y de la coherencia y la adecuación entre las bases teóricas de la investigación y la manera de llevarla a la realidad empírica.

\section{La triangulación en la investigación propia}

\subsection{Las preguntas de investigación y el contexto}

Como nuestro objetivo no es la presentación de toda la investigación ni de sus resultados sino la reflexión sobre la metodología aplicada a ella, nos limitaremos a describir nuestro estudio mediante las preguntas de investigación y la explicación de sus conceptos claves, incluyendo el contexto para pasar a continuación a la reflexión metodológica.

La pregunta principal de nuestra investigación es:

PP: ¿Cómo la didáctica basada en tareas - con ejemplo de las tareas comparativas - influye en el desarrollo de la Competencia Comunicativa Personal (en 
adelante (CP) de los aprendices que estudian con autonomía parcial en el sistema educativo CLIL? (Majewska 2013: 379).

El sistema educativo CLIL se refiere aquí a la sección bilingüe española en un Instituto de secundaria de Polonia, en el que después de un año de estudio intensivo de español (18 horas semanales desde el nivel cero) los estudiantes que estaban en el curso primero (17/18 años de edad) tenían tanto clases de español como lengua extranjera (ELE), como de historia de España, geografía de España y literatura española (asignaturas bilingües) (Tatoj et. al. 2008, Majewska, Tatoj 2009).

Aunque los alumnos investigados formaban una clase entera y fue posible recoger los datos en forma de trabajos escritos y varios cuestionarios de reflexión de cada uno de ellos, la mitad de ellos (14 personas) participó en un programa especial de desarrollo de la autonomía en el aprendizaje, llamado Desarrollo Individual Dirigido. Una vez finalizado el programa, se seleccionaron entre estos últimos a 5 alumnos cuyos datos se presentaron completos (casi 300 páginas de datos) justificando detalladamente la razón de su elección siendo su abundancia una de las razones de no presentar los datos de todos, pero sí, de tenerlos todos en cuenta en la interpretación final de los resultados.

En nuestra investigación las tareas comparativas consistieron en tareas de preparación de textos escritos (y uno oral) de comparaciones interculturales (de las culturas hispana y polaca) a nivel B1/B2, p. ej., la comparación de la Navidad en Polonia con la de España, de dos personas famosas, de dos lugares (regiones), de dos peregrinajes (a Santiago de Compostela y a Częstochowa) o del desempleo en los dos países. Además, escribieron una comparación de dos obras de la literatura española. Las comparaciones interculturales forman parte de los principios de la didáctica en el sistema educativo CLIL, igual que el aprendizaje basado en el discurso, tanto en la comprensión, como en la expresión (Majewska 2013, 2016).

La competencia comunicativa ${ }^{1}$ es un concepto clave en la didáctica de lenguas extranjeras (Wilczyńska, Michońska-Stadnik 2010) por lo cual nunca puede faltar en la investigación. La competencia comunicativa no se analiza como un objetivo educativo común para un grupo de alumnos, sino como un atributo personal, estrechamente ligado a una serie de factores personales y sociales ( $p$. ej. actitudes, convicciones, aspiraciones educativas, planes de

\footnotetext{
${ }^{1}$ La Competencia Comunicativa Personal en nuestra investigación, como se refiere a los estudiantes de CLIL, abarca, no solo las competencias comunicativas de la lengua y las competencias generales del MCER, sino también las competencias clave y las competencias específicas de todas las asignaturas bilingües que cursa el estudiante.
} 
La triangulación múltiple en la investigación-acción con ejemplos de...

desarrollo personal), de ahí la denominación Competencia Comunicativa Personal (Wilczyńska 2002). ${ }^{2}$

La autonomía parcial (o la semiautonomía como la llama W. Wilczyńska 1999) es la autonomía que desarrolla el alumno en colaboración con su profesor en la Zona del Desarrollo Próximo (Vygotsky 1989). En nuestro caso fue todo un programa de desarrollo de la autonomía llamado Desarrollo Individual Dirigido que duró un curso escolar y que empezó con una serie de entrevistas, para a continuación proceder a la elaboración conjunta y a la realización de un plan individualizado del desarrollo lingüístico en español, reflexión profundizada sobre el concepto de la autonomía a base de Portfolio Europeo para las Lenguas, una serie de tareas en las que el estudiante tomaba decisiones, reflexión sobre las tareas realizadas y una entrevista final. El desarrollo de la autonomía es otro principio del aprendizaje en CLIL, primordial, dada la complejidad de los estudios.

Como la pregunta principal es extremadamente compleja, la hemos desglosado en tres preguntas más detalladas (Majewska 2013: 379-381).

PD1: ¿Cómo ha evolucionado la competencia discursiva de los alumnos elegidos en la redacción de textos comparativos (y argumentativos como adicionales)?

PD2: ¿Qué conjunto de factores relacionados con las tareas mismas se atribuye a la evolución (o a su falta) en relación con la PD1?

PD3: ¿Qué conjunto de factores relacionados con la persona del alumno se atribuye a la evolución (o a su falta) en relación con la PD1?

Es casi imposible analizar el desarrollo de la competencia comunicativa en su totalidad, en consecuencia siempre hace falta elegir uno de sus aspectos. En nuestra investigación se ha elegido la competencia discursiva ${ }^{3}$, siendo

2 En nuestra investigación fue fundamental la idea de que "la competencia - a diferencia del conjunto de conocimientos y habilidades preestablecidos para todos - es un conjunto de habilidades $\mathrm{p}$ e $\mathrm{r} \mathrm{s}$ o $\mathrm{n}$ a l e $\mathrm{s}$, que el sujeto ajusta de manera $\mathrm{d}$ i $\mathrm{n}$ á $\mathrm{m}$ i c a a sus objetivos, condiciones y tipos de actividades emprendidas en el marco de sus actitudes generales preferidas formadas bajo la influencia de sus sistemas de valores y experiencias" (Wilczyńska 2002: 74, subrayado de Wilczyńska).

${ }^{3}$ La idea del papel integrador de la competencia discursiva surgió como reacción a los modelos de la competencia comunicativa anteriores al de Celce-Murcia, Dörnyei i Thurrell de 1995, en los que, según estos autores, no se precisaba de forma explícita la relación entre sus componentes. Celce-Murcia y otros relacionan los elementos de la competencia comunicativa (en su modelo son las competencias: lingüística, sociolingüística, accional, discursiva y estratégica) de manera que todos contribuyen por igual a la creación del discurso. En otras palabras, (...) todas las subcompetencias convergen en la producción de textos" (Alonso Belmonte 2016: 556). En el caso de nuestra investigación, teniendo en cuenta el carácter personal de competencia, como otros elementos integradores, además 
esta, según Celce-Murcia (2007) y en nuestra opinión, la central de todas las subcompetencias que forman parte de la competencia comunicativa, por lo tanto la más representativa ya que integra a las demás.

La hipótesis fundamental de la investigación fue la siguiente:

Tendrá lugar el desarrollo de la competencia discursiva (y por consiguiente, de la (CP), pero se notarán diferencias entre los alumnos en su grado y sus aspectos, así como respecto a los factores que hayan decidido sobre esta evolución. En el desarrollo de la CCP de cada alumno influirán una serie de factores, tanto los relacionados con las tareas, como los personales, incluyendo el desarrollo de la autonomía.

El planteamiento tan amplio de la investigación no permite usar la metodología cuantitativa, sino que sugiere sobre todo la cualitativa. El objetivo es obtener una imagen lo más amplia posible, aunque limitada que pueda ayudar a observar el desarrollo de la CCP y entender qué factores posiblemente habrán sido decisivos en el caso de cada una de las 5 personas descritas detalladamente $y$, aunque en bastante menor medida, en el caso de los demás alumnos.

\subsection{La justificación del uso de la triangulación y su tipología en la investigación propia}

La principal razón por la que hemos recurrido a la triangulación es que se ha tratado de una investigación cualitativa, aunque también han tenido en cuenta los elementos cuantitativos de carácter no estadístico (la prueba era demasiado pequeña para recurrir a la estadística).

El fenómeno investigado tenía carácter dinámico, ya que interesaba su desarrollo y no la imagen en un momento en el tiempo. El contexto (el aprendizaje en CLIL) ha sido tratado como sistema dinámico con todos sus rasgos característicos (la sensibilidad hacia las condiciones iniciales, la naturaleza nolinear del desarrollo, la autoorganización, la aparición de estados de atractores y repulsores, la coadaptación y el carácter abierto) (Larsen-Freeman, Cameron 2008, Dörnyei 2009). La explicación del desarrollo de CCP no habría sido posible recurriendo a un solo factor, sino a toda una serie de factores de distinta naturaleza.

En lo que se refiere a la interpretación del progreso (a veces con momentos de retroceso) de los alumnos, nos interesaba no solo analizar los documentos en forma de trabajos de los alumnos, siendo esta la primera perspectiva, sino también, como segunda perspectiva, obtener su opinión sobre el trabajo

de la competencia discursiva, pueden considerarse los procesos cognitivos, afectivos y motivacionales del alumno, que tienen el carácter dinámico (Majewska 2013). 
con las tareas propuestas, su visión de la autonomía (considerada otra posible y deseada causa del desarrollo), su actitud hacia el aprendizaje bilingüe y sus necesidades educativas individuales respecto al español y lo hispano. La tercera perspectiva fue la de 4 profesores que trabajaban en la sección bilingüe, cuyas observaciones servían de confrontación de las declaraciones de la segunda perspectiva (la de los alumnos). Adicionalmente los estudiantes voluntariamente se sometieron a dos tests psicológicos estandarizados, lo que permitió obtener la cuarta perspectiva que ha sido de valor a la hora de interpretar los datos. Además, los datos se analizaron de dos maneras diferentes: la individual (según el desarrollo de cada uno de los alumnos y su interpretación) y desde el punto de vista del fenómeno de la integración de la lengua, los contenidos y los procesos cognitivos en CLIL y sus condicionamientos, dando dos imágenes diferentes, una individual y otra más general.

De lo anterior se desprende que en la investigación ha aparecido la triangulación de fuentes de datos, de investigadores, de metodología y de análisis de los datos obtenidos. Además, los datos han sido obtenidos a lo largo de un curso escolar (triangulación temporal) y en distintos lugares, p. ej. en clase, fuera de clase, durante una excursión escolar (triangulación espacial). Por todo ello se puede calificar la triangulación como múltiple. A continuación se analizarán los procedimientos de cada una de las triangulaciones.

\subsection{La triangulación de fuentes de datos en la investigación}

En la investigación se han recogido dos tipos de datos: (1) de carácter lingüístico y (2) de información sobre las posibles causas y contextos del desarrollo de la CCP.

La fuente principal de información sobre el desarrollo de la competencia discursiva de los alumnos la constituyen los trabajos de los alumnos. La triangulación de datos de carácter lingüístico se ha conseguido gracias a que:

(1) Los textos han sido muchos (todos los alumnos de la clase investigada han escrito obligatoriamente 5 textos comparativos diferentes, han presentado una comparación oral en grupo y han escrito un texto argumentativo).

(2) Los textos han sido preparados a lo largo de muchos meses (triangulación temporal).

(3) Los textos han sido escritos en diferentes contextos espaciales: en clase de ELE, en clase de literatura o en casa (triangulación espacial).

(4) Además de los textos obligatorios, los alumnos han tenido la posibilidad de escribir textos adicionales, lo que muchos han aprovechado para practicar más.

(5) Además de los trabajos escritos, uno ha sido oral. 
(6) Además de los trabajos individuales, uno ha sido grupal.

(7) Los textos se han escrito como trabajos de dos asignaturas diferentes (ELE y literatura española), con dos profesores diferentes, gracias a lo cual se ha podido observar (o no) la posible transferencia de la competencia discursiva y eliminar parcialmente la influencia del investigador.

(8) Además del género discursivo principal (textos comparativos ${ }^{4}$ ), los estudiantes han escrito textos argumentativos, lo que permitió ver el transfer (o su falta) de la competencia discursiva de un género a otro.

Todos los textos comparativos se han analizado con los criterios de operacionalización de la competencia discursiva (anexo). Los criterios de evaluación de la competencia discursiva de los textos argumentativos y los de la comparación oral han sido parecidos a los de los textos comparativos escritos, pero adaptados al género discursivo concreto.

Gracias a la triangulación se ha podido observar la dinámica individual del desarrollo de la competencia discursiva, en unos casos de aumento constante, aunque no en todos los criterios por igual, y en otros casos con retrocesos y vueltas a la subida. Se ha podido observar la transferencia de conocimientos de unos textos a otros, de una asignatura a otra. También se han notado claras influencias en la calidad de trabajo de las preferencias personales, por ejemplo: en un caso por el discurso oral, en otro por la literatura (afición personal), en otro por el arte (también una pasión). Un dato interesante adicional que nos ha sorprendido han sido las elecciones de algunos alumnos, siendo mucho más interesantes y profundas de lo que se podía esperar a estas edades (p. ej. la elección de la comparación del Cid Campeador con el cabaIlero polaco Zawisza Czarny, de los pintores S. Dalí y Z. Beksiński, de la transición a la democracia en Polonia y en España, además de las típicas comparaciones de dos actores o actrices o de dos deportistas).

La triangulación de información sobre las posibles causas y contextos del desarrollo de la CCP se ha conseguido gracias a:

\footnotetext{
${ }^{4}$ La comparación puede entenderse como: operación mental, base para la construcción del sistema de nociones, función académica de la lengua o como género discursivo (puede resultar discutible). Como género, sus rasgos característicos son: el lugar central de los criterios de comparación, la presencia de las diferencias y similitudes entre los fenómenos estudiados y la justificación argumentativa de las mismas por el autor del texto. Suelen escribirse en el ámbito académico (p. ejemplo textos comparativos sobre literatura). Los criterios de comparación no se pueden sustituir por la mera descripción de los dos fenómenos (Majewska 2013).
} 
(1) Entrevistas de carácter semiabierto al principio de la investigación (dos entrevistas, una sobre las actitudes realizada durante la excursión y una sobre el programa del desarrollo individual dirigido de la autonomía efectuado en la escuela, fuera de clases) y al final de la investigación (entrevistas personalizadas sobre el desarrollo de la autonomía y las tareas realizadas, sus actitudes y otros datos de interés). Las de las 5 personas sometidas al análisis individual se han transcrito completas.

(2) Los apuntes de los alumnos en los diarios del Desarrollo Individual Dirigido. Los 14 alumnos cuyos datos completos se han recogido fueron haciendo apuntes durante casi todo el curso escolar. Los diarios completos de las 5 personas elegidas se han adjuntado al estudio.

(3) Tres distintas encuestas de carácter sobre todo abierto de metarreflexión sobre las tareas realizadas (después de escribir tres textos comparativos, después del último y después de la tarea oral).

(4) Los apuntes en el Portfolio Europeo para las Lenguas (Bartczak et al. 2005: 30) completados en una encuesta en la que se han pedido detalles sobre las declaraciones de los alumnos presentadas en el Portfolio.

(5) Otros: observaciones en clase y fuera de clase, conversaciones informales.

Desgraciadamente resultó imposible tomar nota de ellos por falta de tiempo y por desempeñar dos roles a la vez: de profesor y de investigador. Tienen carácter complementario y han servido de orientación para la investigación.

En cuanto al desarrollo de la autonomía, la triangulación ha sido crucial. Los alumnos han rellenado la hoja de la autoevaluación del Portfolio muy rápido dándose muchos puntos, pero cuando en la misma clase se les pidió en el formulario adicional dar ejemplos o precisar las respuestas, muchos empezaron a bajarse los puntos por darse cuenta de que p. ej. ver una película en versión original una vez no es suficiente para decir que se desarrolle la lengua de manera autónoma viendo películas. Otro ejemplo, muchos creían que conocían bien sus puntos fuertes y débiles, sin embargo no fueron capaces de enumerarlos. En cualquier caso se dieron cuenta de que la autonomía contiene muchos aspectos y que ofrece muchas posibilidades distintas de desarrollo.

Nos sorprendió que casi todos los alumnos de la clase que cumplían las condiciones iniciales (por un lado, ir a clase y realizar las tareas de casa sin falta, o sea, aprovechar lo que ofrece el sistema educativo, por otro, no ser verdaderamente autónomo por sí mismo) se presentaran a la entrevista inicial para participar en el programa de Desarrollo Individual Dirigido. Lo interpretamos como prueba (aunque a pequeña escala) del interés de parte de los alumnos por aprender a desarrollar su competencia comunicativa de manera 
autónoma. Como se ha mencionado, la mayoría de ellos trabajó de forma individual todo el año, lo que consideramos un porcentaje alto.

Asimismo nos extrañó que las necesidades de desarrollo individual apenas se repitieran entre tantos alumnos. La conclusión muy importante de carácter adicional respecto a la investigación es que ni siquiera con la cantidad de horas de español y en español en la sección bilingüe (el mayor número de horas de todo el sistema educativo polaco) es posible satisfacer las necesidades individuales de cada alumno. Por lo cual, se confirma la premisa de la necesidad del desarrollo de la autonomía del alumno en el sistema CLIL para que puedan responder ellos mismos a sus necesidades.

Otro dato de interés es que los estudiantes al principio no sabían enumerar muchas de las actividades que podrían hacer sin el profesor. En la entrevista final, casi todos declararon que necesitaban al profesor únicamente para que les indicara materiales de cuya existencia no se hubieran enterado de otra forma y para corregirles los trabajos escritos. Sin problema expusieron varias maneras de desarrollar su CCP ellos mismos. Gracias a la observación, recogida de información sobre la participación en las actividades extraescolares en español, sus preguntas informales, las fuentes de informaciones que habían encontrado y utilizado, los apuntes en sus diarios etc. podemos ver que no han sido meras declaraciones, sino unos comportamientos concretos, variados y frecuentes.

La contribución del desarrollo de la autonomía al desarrollo de la competencia discursiva de los alumnos ha sido directa e indirecta.

Directa, dado que la autonomía se puede definir como la voluntad de tomar decisiones y la responsabilidad por las elecciones respecto a su propio aprendizaje, y en cada tarea hubo un elemento o más a elegir. De los datos recogidos resulta que lo más problemático para los alumnos hasta el final de la investigación fue elegir las fuentes de información adecuadas. Otra contribución directa es que se ofrecieron tareas adicionales de redacción de textos comparativos y argumentativos y varios alumnos aprovecharon esta oportunidad.

Indirecta, ya que cualquier trabajo extra sobre el desarrollo lingüístico en español pudo tener influencia en la calidad de los trabajos investigados.

Los datos de las encuestas de metarreflexión sobre las tareas realizadas, por un lado, han arrojado luz sobre el carácter de las dificultades de los alumnos, sobre su consciencia sobre los rasgos característicos de los géneros discursivos trabajados, sobre el modo de colaboración de los alumnos en la tarea grupal, sobre los motivos de sus elecciones y sus preferencias respecto a las tareas realizadas. Ha sido más fácil entender las posibles causas extralingüísticas del nivel - más alto o más bajo - de algunas tareas en algunos criterios alcanzado por los alumnos. Curiosamente de sus reflexiones se desprende que demasiada libertad de elección de los parámetros de la tarea para muchos resulta 
agobiante, mientras que la elección limitada a pocas opciones para muchos es la ideal, ya que satisface su necesidad de influir en sus propias acciones, y no les hace pasar mucho tiempo dando vueltas para elegir. También se ha apreciado mucho el hecho de que la profesora-investigadora haya dedicado tiempo individualmente a explicar los defectos en la competencia discursiva (no se ha dedicado tiempo adicional a explicar a cada uno sus errores gramaticales o lexicales, ya que la mayoría de los alumnos los suele entender a base de las correcciones marcadas en su redacción, lo que no ocurre con errores de lógica o coherencia/cohesión). Otra observación nuestra es que las tareas de comparación intercultural por exigir mucho tiempo y esfuerzo no se deberían realizar con demasiada frecuencia (parece que la frecuencia propuesta ha sido acertada).

Por otro lado, los alumnos apreciaron mucho el hecho de que les resultara imposible copiar de internet ya que no existían en la red textos parecidos. Este hecho garantizó la autenticidad del trabajo del alumno, exceptuando una posible ayuda por parte del compañero, la cual no se puede considerar del todo negativa.

En conclusión, la elección de las tareas parece ser acertada para observar el desarrollo de la CCP de los alumnos y la variedad de instrumentos de recogida de datos sobre su autonomía parece que han dado datos de fiar.

\subsection{Triangulación de investigadores}

La triangulación de investigadores ha sido relativamente fácil ya que los alumnos investigados tenían clases de español o en español con 4 profesores diferentes incluyendo la investigadora, de los cuales uno era nativo de lengua española. Los profesores les impartían las siguientes asignaturas: Geografía de España, Historia de España, Literatura Española y ELE. Una de las profesoras, además, era la tutora de la clase. La colaboración de todo el equipo ha sido excelente.

El principal instrumento de recogida de datos ha sido en este caso una entrevista semiabierta con todos los alumnos cuyas transcripciones completas se han adjuntado a la memoria. La entrevista tenía como objetivo contrastar las declaraciones de los estudiantes y las observaciones de la investigadora con las observaciones de los demás profesores. En la entrevista los profesores describieron la actitud de los estudiantes respecto a las asignaturas en lengua española, explicaron las tareas adicionales que habían propuesto a los alumnos e indicaron su participación en ellas (autonomía), así como la actividad de los alumnos en sus clases, la manera en que utilizaban las fuentes de información, como organizaban ellos mismos el trabajo (otro aspecto de la autonomía) y su nivel de lengua en discursos orales y escritos. Otra fuente de información han sido los comentarios de los profesores a lo largo del curso. 
Gracias a esta triangulación se han podido confirmar algunos datos respecto a la autonomía de los alumnos y a su actividad, así como la dificultad que encuentran en evaluar las fuentes de información.

Otra observación interesante, que no ha sido fundamental para la investigación pero que valdría la pena comprobar en el futuro a mayor escala, es que la actitud positiva hacia una asignatura (Historia, Geografía o Literatura) en lengua materna parece influir de manera visible en la actitud del estudiante hacia esa misma asignatura en español, mientras que la actitud negativa parece causar que estudiando esa materia en español al estudiante le interese sobre todo desarrollar su nivel de lengua. Es interesante ya que muchas veces los padres dicen que los resultados poco satisfactorios de las asignaturas bilingües se deben a dificultades en estudiar en lengua extranjera y parece que no es lo decisivo. Es un ejemplo de que la metodología cualitativa puede hacernos descubrir datos que parecen marginales en la investigación que se lleva a cabo, pero que pueden inspirar las futuras investigaciones.

Otra manera de asegurar la triangulación de investigadores fue la colaboración con un psicólogo profesional, lo que se detallará en el apartado dedicado a la triangulación de métodos.

\subsection{Triangulación de métodos}

En la investigación, además de los métodos y técnicas propias de la pedagogía (y didáctica de lenguas extranjeras) se han utilizado dos tests psicológicos estandarizados. En Polonia solo los psicólogos profesionales tienen acceso a ellos. Por esa razón y para estar segura de la elección de las herramientas de investigación, la investigadora colaboró con un profesor universitario de psicología con el título entonces de doctor (actualmente profesor catedrático). Fue él quien aconsejó el uso de los dos tests y el que se responsabilizó por su realización e interpretación, garantizando su calidad.

El primer test fue el Inventario de Personalidad NEO Ffi de Costa y McCrae (Zawadzki et al. 1998), adaptado a las condiciones polacas y a la edad de los alumnos. El test mide los 5 rasgos fundamentales de la personalidad: la extroversión (extraversion), apertura a experiencias nuevas (openness to experience), neuroticismo (neuroticism), meticulosidad (conscientiousness) y afabilidad (agreeableness). Se sometieron a la prueba todos los estudiantes de la clase investigada, con permiso de sus padres.

La utilidad de este instrumento para la explicación de los progresos (o su falta) y las actitudes de los alumnos ha sido realmente importante. La apertura a experiencias nuevas de una de los 5 alumnos analizados individual mente ha explicado su actitud muy abierta hacia distintas propuestas de actividades 
extraescolares y hacia el programa del Desarrollo Personal Dirigido de la autonomía, al igual que su enfoque hacia uno de los textos comparativos en el que la alumna ha ampliado el tema (la comparación de tres fenómenos de tres culturas en lugar de dos: la comparación de la historia de la canción "L’Estaca" de Lluis Llach con sus versiones polaca y cubana), ha sobrepasado con diferencia el límite de palabras y ha escrito un artículo de prensa en lugar de un texto comparativo (la diferencia sobre todo se refleja en el estilo). Ella misma subrayó que estaba dispuesta a recibir peor nota por eso ya que lo más importante para ella era expresar lo que quería y cómo lo quería hacer. La máxima meticulosidad en el caso de otra alumna explica la mayor cantidad de trabajos entregados por ella (escribió todos los textos adicionales). La alta extraversión junto con la alta afabilidad explican la preferencia por la presentación oral en grupo. En general, este instrumento ha permitido interpretar mejor la actitud de los alumnos hacia las tareas y hacia el aprendizaje autónomo.

Sin embargo, otro instrumento sugerido, es decir, el cuestionario de lugar de control para los jóvenes de 13-17 años (Krasowicz, Kurzyp-Wojarska 1990) no ha ayudado en la interpretación de los resultados. Una de las razones, en nuestra opinión, fue que el test era relativamente viejo y las preguntas no reflejaban bien la realidad de los jóvenes de las últimas generaciones que viven mucho en el mundo digital, lo que se pudo notar por sus gestos en el momento de leer las preguntas. Por ello solo se mencionó en el estudio el hecho de realizarlo en lugar de servirse de sus resultados.

Un valor añadido de recurrir a los tests psicológicos es que los estudiantes recibieron los resultados y su interpretación en las direcciones de sus correos electrónicos, lo que muchos han apreciado.

\subsection{La triangulación del análisis de los resultados}

El último tipo de triangulación aplicada en nuestro estudio es la del análisis de resultados. Se han analizado los resultados desde dos puntos de vista.

El primero es el desarrollo individual de la competencia discursiva de cada uno de los 5 alumnos elegidos. En cada caso primero se ha analizado el desarrollo de la competencia discursiva a base del análisis de los textos escritos y la transcripción de la presentación oral. Después se han analizado los factores que habrán condicionado el progreso del alumno, los relacionados con las tareas y los relacionados con el alumno como persona.

El segundo nivel del análisis presentado en la memoria tiene carácter más general. Por una parte se intentan generalizar los resultados individuales, ampliando la visión con los resultados de los demás alumnos de la clase sobre los que se recogieron los datos empíricos más o menos completos, constituyendo 
estos un fondo para la interpretación de los fenómenos. Por otra parte, la generalización consistía en un intento de referir los resultados al modelo teórico de la integración de la lengua, los contenidos y los procesos cognitivos de orden superior, presentado en la parte teórica de la memoria. Los resultados han sido organizadas en torno a las tres preguntas detalladas de la investigación empírica (véase apartado 3.1.).

En el caso de PD1. se ha intentado responder a las siguientes preguntas: ¿Ha tenido lugar el desarrollo de la competencia discursiva? ¿Cuál ha sido la dinámica general del desarrollo? ¿Cuál ha sido la dinámica del desarrollo en cuanto a los criterios detallados de la evaluación de la competencia discursiva en los textos comparativos? ¿Cuál ha sido la escala y el alcance del progreso en los dos géneros discursivos? ¿Depende la dinámica del progreso del género discursivo? ¿Ha tenido lugar una transferencia de la competencia discursiva entre los géneros discursivos y de la asignatura ELE a la asignatura Literatura?

Se han resumido los resultados presentando la competencia discursiva como un reto individual.

En lo que se refiere a PD2 el análisis ha girado en torno a las siguientes cuestiones: ¿La tarea (redactar textos comparativos interculturales) ha cumplido los requisitos de la Tarea Basada en el Procesamiento Profundo de Información? ¿Se ha confirmado la hipótesis del papel central del discurso (y del género discursivo) y de la producción lingüística en CLIL? ¿En qué se ha manifestado el valor cognitivo y la multiperspectividad de la tarea? ¿Han permitido las tareas desarrollar los conocimientos y las habilidades de las asignaturas bilingües (Literatura, Historia, Geografía) conformes a la modalidad de la clase? ¿Ha desarrollado la tarea el pensamiento de orden superior y/o el pensamiento crítico? ¿Qué importancia ha tenido el elemento de la elección en comparación con el de la determinación por parte del profesor de los parámetros de la tarea? ¿Ha permitido la tarea desarrollar las competencias clave? ¿Qué importancia ha tenido la metarreflexión sobre las tareas comparativas? ¿Cuál ha sido la relación entre la imagen del propio desarrollo (autoevaluación) respecto a la redacción de textos comparativos y a la motivación al desarrollo? ¿Qué importancia han tenido las estrategias individuales de la preparación de los textos comparativos? ¿Qué importancia ha tenido su relación afectiva con la tarea de comparación?

Se han recapitulado los resultados conforme a la teoría de los sistemas dinámicos indicando los atractores del desarrollo del sistema relacionados con la tarea.

En lo que refiere a la PD3 el análisis pretendió dar respuestas a las siguientes cuestiones: ¿Qué condicionamientos de naturaleza organizativa se han atribuido al desarrollo de la competencia discursiva? ¿Hasta qué punto ha tenido importancia la convergencia entre las decisiones tomadas y los planes 
personales de autoformación? ¿Qué importancia ha tenido la actitud hacia el aprendizaje de las asignaturas bilingües y hacia CLIL? ¿Qué otras actividades autónomas han tenido lugar y qué importancia han tenido? ¿Es posible constatar la consciencia de la autonomía y hasta qué punto tiene carácter objetivo? Cuáles son los valores de la autonomía según los alumnos? ¿Qué importancia ha tenido cada uno de los cinco rasgos de personalidad en el desarrollo del alumno?

Se han concluido los resultados presentando los atractores del desarrollo del sistema relacionados con el alumno como persona y de organización de su trabajo.

Como conclusiones de toda la investigación se ha intentado dar respuesta a la pregunta principal, indicando con claridad la necesidad de plantear más trabajo de campo sobre el desarrollo de la CCP de los alumnos en CLIL.

\section{Conclusiones}

La triangulación es una estrategia fundamental en la metodología cualitativa, incluyendo la investigación-acción. Es la estrategia primordial para asegurar su calidad en sus diferentes aspectos.

El valor más importante de la triangulación es, a nuestro modo de ver, ofrecer nuevas perspectivas y nuevas imágenes de un mismo fenómeno investigado. La premisa en la que se basa este hecho es que no hay una sola interpretación correcta de la realidad social, sino que sean posibles interpretaciones y visiones alternativas, muchas de ellas bien justificadas y enriquecedoras.

La triangulación tiene principalmente dos funciones.

La primera es contrastar y comparar los datos obtenidos de una fuente de datos con los de otras buscando similitudes y divergencias. Encontrar datos parecidos nos permite confirmar los datos obtenidos previamente. Los datos divergentes nos hacen reflexionar sobre sus posibles causas y consecuencias. En primer lugar, volvemos a analizar la adecuación del instrumento y tomamos en consideración su eventual revisión o cambio. Este ha sido el caso de uno de los tests psicológicos elegidos, cuyos resultados no se han tenido en cuenta a la hora de interpretar los resultados. No obstante, no siempre es una buena solución, ya que los instrumentos han podido ser bien elegidos, pero por la influencia de ciertos factores (p. ej. influencia del contexto nuevo o desconocido) nos pueden mostrar una perspectiva nueva que enriquece nuestro conocimiento de la realidad investigada.

La segunda función es la de ofrecernos la posibilidad de percibir fenómenos en su complejidad y dinamismo. Gracias a las imágenes que recibimos de distintos investigadores, en diferentes momentos de tiempo, en diferentes lugares, de las diferentes fuentes y métodos, nos acercamos a una visión más completa y por eso más verdadera que en el caso del uso de un método o instrumento usado en un momento e interpretado por una persona. 
La triangulación de métodos y fuentes de datos nos permite, por un lado, ir más allá de las meras declaraciones de personas y por otro lado, gracias a estas declaraciones incluir la visión subjetiva, introspectiva a la observación objetiva y al análisis de documentos. La triangulación de investigadores posibilita evitar el sesgo.

El tipo de triangulación más difícil y menos utilizado es el de teorías, el más usado, el de fuentes de datos. También es cada vez más frecuente la triangulación que consiste en recurrir a la metodología cualitativa y cuantitativa en un mismo estudio, aprovechando lo mejor que ofrece cada una de ellas.

La triangulación más aconsejable y la que más calidad puede aportar a la investigación es la triangulación múltiple (Ilamada también multimétodo) que consiste en usar dos o más tipos de triangulación posible. Es especialmente útil en el caso de investigar sistemas dinámicos, en los que influyen muchos factores diferentes y de distinta fuerza en cada caso individual, muy dependientes del contexto y por todo ello muy complejos, imposibles de reducir a una simple dependencia de tipo causa-efecto.

El aprendizaje en una sección bilingüe (sistema educativo tipo CLIL) es un ejemplo de un contexto extremadamente complejo y, en el caso de Polonia, todavía no muy extendido, además, sometido a constantes cambios. Incluso si elegimos bien el objeto de investigación - que ha sido en nuestro caso el desarrollo de la competencia discursiva en el género discursivo bien fundamentado por el contexto (textos comparativos interculturales basados en distintas fuentes) y por los objetivos de la enseñanza bilingüe (integración de la lengua, los contenidos y los procesos cognitivos de orden superior) - es imposible interpretar los resultados sin tener en cuenta las actitudes del alumno, sus preferencias, dificultades, su aprendizaje autónomo en los diferentes aspectos que incluye, y otros factores de índole psicológica, social o behavioral. Por eso hemos optado por centrarnos en su Competencia Comunicativa Personal, subrayando el adjetivo personal. Esa es también la razón de incluir los tests de personalidad en la interpretación de los resultados.

Tal y como es de esperar, gracias a la triangulación, además de los resultados más o menos previsibles, han aparecido datos de carácter adicional, pero importantes desde el punto de vista de la organización de la enseñanza en las secciones bilingües. A modo de ejemplo, resulta utópico esperar satisfacer las necesidades de los alumnos incluso en las secciones bilingües, que cuentan con mayor número de horas en el sistema educativo siendo estas muy diversas y poco repetitivas. Del mismo modo dejar a los alumnos total libertad en cuanto a la elección de los parámetros de la tarea de redacción resulta poco cómodo para muchos, que prefieren elegir entre contadas opciones y no con libertad absoluta. Estas observaciones pueden constituir un punto de partida para determinar posibles objetos de investigaciones futuras. 


\section{BIBLIOGRAFÍA}

Aguilar S., Barroso J. (2015), La triangulación de datos como estrategia en investigación educativa. (En) "Píxel-Bit. Revista de Medios y Comunicación", n 47, pp. 73-88.

Aleksandrzak M., (2004), Metodologia action research $w$ badaniach nad autonomiq ucznia. (En) Pawlak M. (red.). Autonomia w nauce języka obcego. Poznań-Kalisz: Wydział Pedagogiczno-Artystyczny UAM w Poznaniu. pp. 111-124.

Alonso Belmonte I. (2016), La subcompetencia discursiva. (En) "Vademécum para la Formación de Profesores" Sánchez Lobato, J., Santos Gargallo, I., Alcobendas (Madrid): SGEL pp. 553-572.

Alzás García T., Casa García L. M., Luengo González R., Torres Carvalho J. L., Verissimo Catarreira S. (2016). Revisión metodológica de la triangulación como estrategia de investigación. (En) "Investigación Cualitativa en Ciencias Sociales" volumen 3, pp. 639-648.

Arias Valencia M.M., (2000), La triangulación metodológica: sus principios, alcances y limitaciones. (En) "Investigación y Educación en Enfermería", vol. XVIII, $n^{\circ} 1$, pp. 13-26.

Bartczak E., Lis Z., Marciniak I., Pawlak M., (2005). Europejskie portfolio językowe dla uczniów szkół ponadgimnazjalnych i studentów. Warszawa: CODN.

Burns A., (1999), Collaborative Action Research for English Language Teachers. Cambridge: CUP.

Celce-Murcia, M., (2007). Rethinking the Role of Comminicative Competence in Language Teaching. (En) Alcón Soler, E., Safont Jordá, M.P. (reds.)., Intercultural Language Use and Language Learning. Springer Science nad Business Media B.V. Sin indicar el lugar de la edición. pp. 41-57.

Denzin N.K., Lincoln Y.S. (red.). (2009). Metody badań jakościowych. Volumen 1. Warszawa: Wydawnictwa Naukowe PWN.

Dörnyei Z. (2009). The Psychology of SLA. Oxford: Oxford University Press. Flick U., (2007), Introducción a la investigación cualitativa. Madrid: Morata.

Hornowska E., Brzezińska A.I., Kaliszewska-Czeremska K., Appelt K., Rawecka J., Bujacz A. (2012), Paradoksalny efekt triangulacji? (En) „Edukacja”, n 4(120), pp. 72-83.

Kemmis, S., McTaggart R., (2009), Uczestniczq̨ce badania interwencyjne. Dziatanie komunikacyjne i sfera publiczna. (En) Denzin N.K., Lincoln Y.S. (reds.), Metody badań jakościowych. Tom 1 Warszawa: Wydawnictwo Naukowe PWN. pp. 775-832.

Konarzewski K., (2000), Jak uprawiać badania oświatowe. Metodologia praktyczna. Warszawa: WSiP. 
Krasowicz G., Kurzyp-Bojarska A., (1990). Kwestionariusz do badania poczucia kontroli (KBPK). Warszawa: Polskie Towarzystwo Psychologiczne.

Larsen-Freeman, D., Cameron, L., (2008), Complex Systems and Apllied Linguistics. Oxford: OUP.

Majewska R., Tatoj C., (2009), Sprawozdanie z badań jakościowych prowadzonych w klasach dwujęzycznych z językiem hiszpańskim w polskich gimnazjach $i$ liceach. (En) Neofilolog $n^{\circ} 33,227-238$.

Majewska R., (2013), Zintegrowane kształcenie przedmiotowo-językowe. Dydaktyka zadaniowa a rozwój osobistej kompetencji komunikacyjnej $w$ warunkach półautonomii. Tesis doctoral no publicada, vols. 1 y 2 . Poznań: Universidad Adam Mickiewicz.

Majewska R., (2016), Integrujqce zasady dydaktyczne w systemie CLIL. (En) Jaroszewska A., Karpeta-Peć B., Smuk M., Sobańska J., Sujecka-Zając J. (reds.). Wielojęzyczność i międzykulturowość na lekcji języka obcego między teorią a praktyką nauczania.

Palka S. (2006), Metodologia.Badania. Praktyka pedagogiczna. Gdańsk: GWP. Pilch T., Bauman T. (2001), Zasady badań pedagogicznych. Warszawa: Wydawnictwo Pedagogiczne „Żak”.

Silverman D. (2008), Prowadzenie badań jakościowych. Warszawa: Wydawnictwo Naukowe PWN.

Szymańska M., Ciechowska M., Pieróg K., Gołąb S. (2018), Badania w działaniu w praktyce pedagogicznej. Kraków: Wydawnictwa Naukowe Akademii Ignatianum w Krakowie.

Tatoj C., Majewska R., Spychała M., Zając M., Piech E., (2008), Raport ewaluacyjny. Sekcje dwujęzyczne z językiem hiszpańskim w Polsce. Warszawa: CODN.

Urbaniak-Zając D. (2018), O łq̨czeniu badań ilościowych i jakościowych - oczekiwania i wq̨tpliwości. (En) „Przegląd Badań Edukacyjnych”, n² 26, pp. 121-138.

Vygotsky, L. S., (1989). Myślenie i mowa. Warszawa: PWN.

Wilczyńska W., (1999), Uczyć się czy być nauczanym? Warszawa: Wydawnictwo Naukowe PWN.

Wilczyńska W. (2002), Podmiotowość i autonomia jako wyznaczniki osobistej kompetencji komunikacyjnej. (En) Wilczyńska W. (red.), Doskonalenie się w komunikacji ustnej. Poznań: Wydawnictwo Naukowe PWN, pp. 51-68.

Wilczyńska W., Michońska-Stadnik A., (2010), Metodologia badań w glottodydaktyce. Wprowadzenie. Kraków: Avalon.

Wiśniewska D. (2009), Badanie w działaniu jako metoda badawcza $w$ dydaktyce języków obcych. (En) „Neofilolog” n 32, pp. 115-126.

Zawadzki B., Strelau J., Szczepaniak P., Śliwińska M., (1998). Inwentarz osobowości NEO-FFI Costy i McCrae. Adaptacja polska. Podręcznik. Warszawa: Pracownia Testów Psychologicznych Polskiego Towarzystwa Psychologicznego. 


\section{Anexo}

\section{“Criterios de evaluación de la competencia discursiva de textos comparativos}

1. La longitud del texto respeta el límite, determinado por la cantidad de palabras. El contenido es exhaustivo. El texto no incluye elementos superfluos.

2. El texto cumple las condiciones de coherencia respecto a la estructura propios de un texto comparativo. Su composición es correcta (tres variantes del desarrollo del texto: (1) en cada párrafo otro criterio de comparación, (2) un párrafo trata de las diferencias y otro de las similitudes; (3) la descripción de los dos fenómenos y en la conclusión un resumen de las diferencias y similitudes según los criterios de comparación. Los criterios de comparación se perciben claramente del texto ya que contiene tanto las similitudes como las diferencias. Incluye asimismo la introducción y la conclusión y una división en párrafos clara, tanto lógica como gráficamente.

3. El texto cumple con las condiciones de cohesión y coherencia propias del texto comparativo en el aspecto del uso correcto de los conectores discursivos (a nivel micro y macrotextual) y/o otros recursos lingüísticos que expresan comparación (sobre todo a nivel semántico, p. ej. aumentar, disminuir, alcanzar un nivel parecido).

4. El texto cumple con las condiciones de cohesión y coherencia propias del texto comparativo en el aspecto del uso correcto de los conectores discursivos (a nivel micro y macrotextual) no comparativos.

5. El texto no incluye errores chocantes de falta de coherencia estilística (p. ej. en cuanto al registro) o lógica (p. ej. fragmentos incomprensibles, ambiguos, errores resultantes de la falta de una parte de información, falta de determinación clara del sujeto), tampoco incluye los errores gramaticales o lexicales globales que imposibiliten o dificulten considerablemente la comprensión del texto".

(Majewska 2013 vol. 2: 131) 